\title{
An Improved Machine Learning-Based Short Message Service Spam Detection System
}

\author{
Odukoya Oluwatoyin \\ Department of Computer Science and Engineering, Obafemi Awolowo University, Ile-Ife, Nigeria \\ E-mail: olagun12@yahoo.com \\ Akinyemi Bodunde, Gooding Titus and Aderounmu Ganiyu \\ Department of Computer Science and Engineering, Obafemi Awolowo University, Ile-Ife, Nigeria \\ E-mail: bakinyemi@oauife.edu.ng,gooding@gmail.com, gaderoun@oauife.edu.ng
}

Received: 12 November 2019; Accepted: 27 November 2019; Published: 08 December 2019

\begin{abstract}
The use of Short Message Services (SMS) as a mechanism of communication has resulted to loss of sensitive information such as credit card details, medical information and bank account details (user name and password). Several Machine learning-based approaches have been proposed to address this problem, but they are still unable to detect modified SMS spam messages more accurately. Thus, in this research, a stack- ensemble of four machine learning algorithms consisting of Random Forest (RF), Logistic Regression (LR), Multilayer Perceptron (MLP), and Support Vector Machine (SVM), were employed to detect more accurately SMS spams. The simulation was carried out using Python Scikit- learn tools. The performance evaluation of the proposed model was carried out by benchmarking it with an existing model. The evaluation results showed that the proposed model has an increase of $3.03 \%$ of accuracy, $8.94 \%$ of Recall, $2.17 \%$ of F-measure; and a decrease of $4.55 \%$ of Precision over the existing model. This indicates that the proposed model reduces the false alarm rate and thus detects spams more accurately. In conclusion, the ensemble method performed better than any individual algorithms and can be adopted by the Network service providers for better Quality of Service.
\end{abstract}

Index Terms-Short Message Service (SMS), Spam Detection, Ensemble method, Machine Learning.

\section{INTRODUCTION}

Advancement in technology has made end-users to access their emails, surf the World Wide Web, make video and voice calls, use text chatting, make a medical appointment, gaming and more through their smartphones. The purposes of SMS spam are advertisement and marketing of various products, sending political issues, spreading inappropriate adult content and

Internet offers and phishing attack among others, hence spam SMS flooding has become a serious problem all over the world. SMS spamming gained popularity over other spamming approaches like email and Twitter, due to the increasing popularity of SMS communication. Mobile devices now contain personal and confidential information such as credit card numbers, contact lists, emails, medical records and other sensitive documents. The issue of SMS spam can be attributed to limited resources and processing power and lack of knowledge and consciousness to end-users regarding security threats. The aforementioned reasons make mobiles very eyecatching to cyber-attacks. Hackers can utilize the compromised mobiles to make calls to premium numbers without the end-users' permission, stealing contact data, or participating in botnet activities [1]. The exchange of SMS among different mobile phones is very suitable and regularly used for communication on a day to day basis. Then, the number of spam SMS messages is growing. In 2012, there were 350,000 variants of SMS spam globally [2]. SMS has been considered a serious security threat since the early 2000s [3]. For instance, hackers can send phishing attacks to collect confidential information or launch other types of attacks. The risk of SMS spam could lead to operational or financial loss. It is getting easier to attack end-users through SMS than emails, since the mail service is more secure, and more effective email spam filter has been developed and installed by mail service providers and users. In this light, it is not the case of SMS spam. It is a challenging task since SMS messages have limited sizes (160 characters long) which means less statistically-distinguishing information. Several methods have been investigated to detect SMS spam, including $\mathrm{H} 2 \mathrm{O}$ approaches [4]. In this light, the accuracy is still relatively low and further research is required to investigate new features and new ways of tackling this. In this research work, several feature sets and their impact on four machine learning algorithms were studied. The rest of this paper is organized as follows. Section 2 covers the literature review while section 3 describes the methodology and presents the proposed architecture, section 4 discusses the performance metrics and section 5 discusses the results. Finally, Section 6 concludes the paper. 


\section{RELATED WORKS}

There has been quite a lot of work done in the area of SMS spam detection. A new classifier based on the $\mathrm{H} 2 \mathrm{O}$ platform was developed in [4]. The $\mathrm{H} 2 \mathrm{O}$ framework was used for evaluating the accuracy, precision, recall, fmeasure to classify unseen data by using machine learning algorithms. The framework performed better than other frameworks such as Weka, it was used to determine the best features that will be used to improve the SMS Spam detection process. The limitation of the work indicated that other Machine learning (ML) algorithms needed to be added [4]. A review was done in [5] to filter SMS spam using CDA algorithms, KNN and the large cellular network method to measure accuracy, strength and weakness. A comparative review was carried out on various ML algorithms and a systematic search for a proper publisher in the field (study selection, Information Sources, data collection Process and comparison criterion). The work shows that an intensive survey was done to present an accurate result of different text classifier on different datasets for spam filtering. The result of the research showed that lack of public and real datasets, and the low number of features that were extracted per message could hinder the accuracy of the extraction. A proposed approach to filter spam SMS using ML algorithms was proposed in [6]. Datasets were used as features of the experiments. Features were extracted from messages (HAM and SPAM) to create a feature vector. The approach performed better with Random Forest classification algorithms in terms of high accuracy. It achieved the aim of classifying spam SMS message, but there is a need to compare ML algorithms to achieve the desired goal [6]. A systematic literature review on SMS spam detection techniques was performed in [7]. The researcher reviewed the available published research works from 2006 to 2016. Performance comparison of the studied literature was carried out. None of the studies solved the challenges of SMS spam detection of regional contents and shortcut words [7]. A proposal for an appropriate preprocessing method of vector representations and classifiers to find the best model. The proposed method was accomplished using preprocessing step, Vector Representation and Classifiers (Naïve Bayes).The system uses a simple rule to detect spam message by catching a special tag in their content. To achieve better result there is a need to enlarge the corpus to provide more data for the system. Filtering system was used to address the problems of mobile SMS spam; however performance of spam detection is very important and must reach an adequate level by making certain adaptation on filtering techniques [8]. The research in [8] founded on increasing the performance of SMS spam detection by applying the same filter used in email spam filters which achieved the highest performance. Two datasets were used for testing, one for English and another for Spanish. English dataset consists of 1002 hams and 82 spams, while Spanish contains 1157 legitimate and 199 spams. Most of the machine learning algorithms that were applied in the vector representation of messages used certain features such as words, lowercase words, bigram and trigram of characters and words bigrams. Email filtering algorithms became underperformed when used with SMS spam, this is as a result of different reasons: messages with limited features, there was no real database for SMS spam, the informal language of the messages and the short length of it [9].The survey provided a detail summary of spam SMS filtering techniques and algorithms which will help to overcome the problem of SMS spam. The research only focuses on the review of other work in the area of spam SMS filtering techniques using data mining. No system was developed to address the main purpose of the paper [10]. A hybrid system of SMS classification to detect spam or ham, using naïve Bayes classifier and apriority algorithms was proposed. It better contributed to accuracy than the state of the art method of classifying text. A categorization system that integrates association rule mining with the classification problem was built. The categorization system which was based on naïve Bayes filter SMS spam messages based on Bayesian learning and sender blacklisting mechanism was developed, it uses crowd sourcing to keep itself updated. The system was used to test the performance of the email spam filter on Korean, English and Apriority algorithms was based on logic but the result was depended on dataset, significant improvement of state of the art algorithms was depicted. Although the system outperforms the state of the art, but a part of the training of the system required little time than the state of the art. The system slightly took more time [11]. A mobile based system called SMS Assasin that can, and Spanish dataset. A set of design goal was presented, which act as guidelines to design a mobile-based SMS spam filter. A mobile-based SMS spam detection system was implemented; it was done using python S60 on a Symbian platform [12].

Therefore, in this study an attempt was made to provide an SMS spam detection model that will reduce the amount of spam SMS with low false alarm rate.

\section{METHODOLOGY}

In this study, the methodology employed to achieve the objectives are as follows:

\section{A. Conceptual Model Description}

The proposed system architecture as shown in Fig. 1 depicts various phases of SMS spam detection at both training and testing phases. The detailed of the phases are as follows:

- The pre-processing phases consist of steps such as tokenization, removal of stop words, stemming, lemmatization and cleansing of the dataset.

- The feature selection phase is introduced to reduce the dataset and number of features; this was achieved by using consistency-based methods.

- The dataset was then split into both training and testing datasets.

- The training dataset was subjected to classification 
with the ensemble of algorithms.

- The results of the classification were then validated using evaluation metrics such as accuracy, precision, f-measure and recall. The results of the classification are the output of SMS spam detection.

\section{SMS Dataset collection}

In this research, the University of California Irvine (UCI) Machine Learning repository dataset [15] was used. The dataset is a collection of SMS data that has been sent by users of mobile devices. The SMS corpus was marked at the sentence level. Two classes of polarity (both ham and spam) were considered in this research work. The datasets consist of 6250 text messages classified as ham and spam messages, with 1562 spam messages and 4688 ham messages. The dataset was saved in a CSV file format where each line represents one message; the line consists of the label of a message and text string. Table 1 shows examples of messages in the dataset.

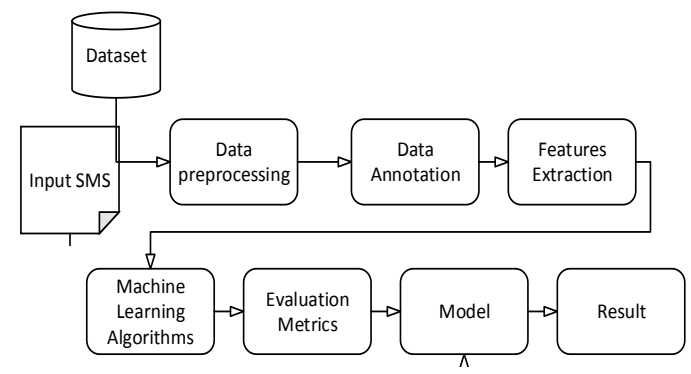

Fig.1. SMS Spam Detection System

Table 1. Examples of Messages in the Dataset

\begin{tabular}{|c|c|}
\hline \multicolumn{2}{|r|}{$\begin{array}{l}\text { Example of ham and spam messages in UCI } \\
\text { dataset and the some of the Nigeria Mobile device user }\end{array}$} \\
\hline Ham & $\begin{array}{l}\text { Have a safe trip to Nigeria. Wish you happiness } \\
\text { and very soon company to share moments with }\end{array}$ \\
\hline Ham & 'Wen did you get so spiritual and deep. Thatl's great' \\
\hline Ham & 'U still havent got urself a jacket ah?', \\
\hline Spam & $\begin{array}{l}\text { "IMPORTANT - You could be entitled up to } £ 3,160 \\
\text { in compensation from mis-sold PPI on a credit card } \\
\text { or loan. Please reply PPI for info or STOP to opt } \\
\text { out. }\end{array}$ \\
\hline Spam & $\begin{array}{l}\text { Dear customer your ATM ACC...NT has been } \\
\text { block due to BVN system error, Quickly call our } \\
\text { Customer Care line } 088031314841 \text { to reactivate } \\
\text { Under } 24 \text { hour }\end{array}$ \\
\hline Spam & $\begin{array}{l}\text { U are a winner of our daily raffle to claim your } \\
\text { prize click on the link 'XXXMobileMovieClub: }\end{array}$ \\
\hline
\end{tabular}

\section{Preprocessing}

The following preprocessing techniques were employed to remove irregularities and inconsistencies from the dataset collected, to capture the problem being addressed. In the preprocessing phase, a feature engineering technique was applied to the dataset, to enable the machine learning algorithm to produce accurate results. The dataset was processed using techniques such as tokenization and stop word removal and cleaned before entering them into the algorithm. Hence, the work engaged the use of data analysis, iterations, and examination for the performance of the classifier. Data was in a text format after processing and for this work, a CSV file format dataset was used. The python natural language processing tool was used for preprocessing the dataset. The pre-processing procedures are as follows:

\section{a. Tokenization}

For the processing of the SMS dataset, words that constitute a string of character were classified. This was done because the meaning of the text depends on the relations of the word in the text. Tokenization is the process of extracting words/ data in a message[16], it also helps in the process of chopping data up into pieces, called tokens, perhaps discarding certain characters, for example, punctuation and the total number of characters. It is frequently applied in the detection of attacks. The framework used for tokenization of the SMS dataset was the Natural Language Tool Kit (NLTK), in NLTK, the method tokenized_xample (text) was used to split the sentences into words. The output of the tokenization was converted to a data frame for better text understanding in machine learning application. In this study, the tokenized data from the dataset is depicted in Fig. 2.

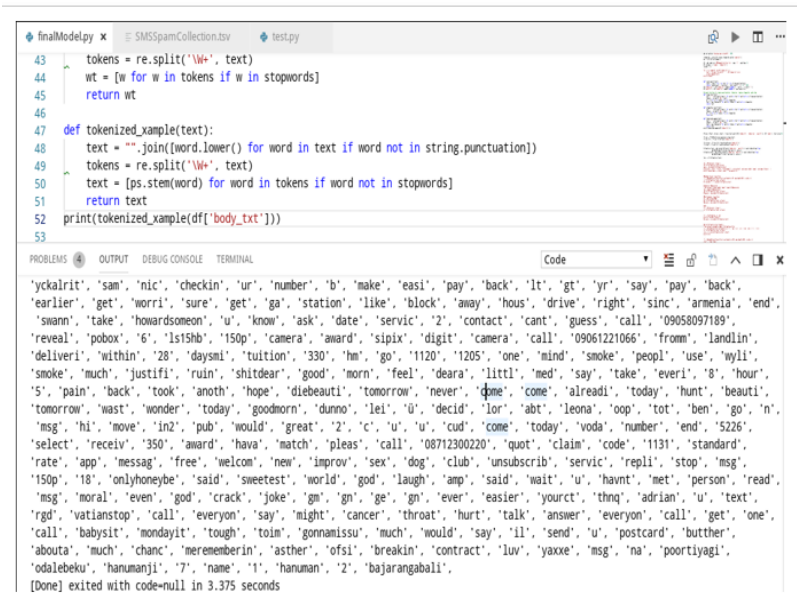

Fig.2. Tokenized dataset

\section{b. Removal of Stopwords}

One of the common data processing steps in NLP is stopword removal. The objective was to remove all common words that occur more frequently in the dataset [17]. Stopwords are some common words in the text messages that have no or little meaning, for example; you, me, a, him, etc. while carrying on experiment of text analysis, some words have features and are weighty in the text message, they are considered as features, whereas some words do not contribute at all, are considered as stopwords. Usually, articles and pronoun are normally classified as stop words. Fig. 3 shows the example of stopwords in the dataset. For removal of stopwords from the SMS dataset, the natural language tool kit (NLTK) was used. In NLTK, the method stopwords_result (text), was used to remove words that do not contribute to future operation in the sentences. 


\section{c. Annotation of the SMS Dataset}

The process of adding metadata information to the text to augment a computer capability to perform NLP is what is referred to as annotation. Features shown in Table 2[4] were used to encode the specific phenomenon to capture the desired behavior of the algorithms that were trained. Metadata was added to each text in the dataset to signify whether the message is spam or ham. In the process, 1 was added for spam and 0 for ham message.

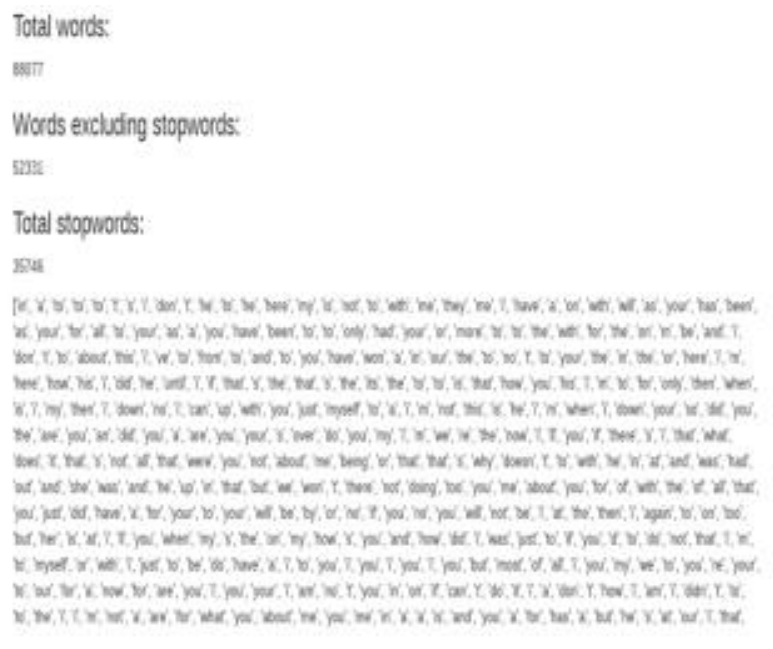

Fig.3. Stopword in the dataset

Table 2. Description of features

\begin{tabular}{|c|c|c|}
\hline $\mathbf{S} / \mathbf{N}$ & $\begin{array}{l}\text { Features } \\
\text { description }\end{array}$ & Features criteria \\
\hline$\# 1$ & $\begin{array}{l}\text { Message Length } \\
(\mathrm{ML})\end{array}$ & $\begin{array}{l}\text { The number of characters } \\
\text { in the message }\end{array}$ \\
\hline \#2 & $\begin{array}{l}\text { Number of Words } \\
(\mathrm{NW})\end{array}$ & $\begin{array}{l}\text { The number of words in } \\
\text { message, usually spam } \\
\text { messages contains } \\
\text { large number of words }\end{array}$ \\
\hline \#3 & $\begin{array}{l}\text { Ratio of Number of } \\
\text { Words with length } \\
\text { less than three } \\
\text { (RNW3) }\end{array}$ & $\begin{array}{l}\text { Number of words less than } \\
3 \text { (NW3) over to the total } \\
\text { number of } \\
\text { words (NW) }\end{array}$ \\
\hline \#4 & $\begin{array}{l}\text { Ratio of Number of } \\
\text { Capital (RCW) }\end{array}$ & $\begin{array}{l}\text { Number of Capital Words } \\
(\mathrm{CW}) \text { over total number of } \\
\text { words (NW) }\end{array}$ \\
\hline \#5 & $\begin{array}{l}\text { Ratio of } \\
\text { Alphanumeric } \\
\text { Characters (RAC) }\end{array}$ & $\begin{array}{l}\text { Number of Alphanumeric } \\
\text { Characters over Message } \\
\text { Length (ML) }\end{array}$ \\
\hline \#6 & $\begin{array}{l}\text { Ratio of Special } \\
\text { Characters (RSC) } \\
\text { such as “*, } \\
,+,=, \%, \$, @,-, \downarrow, /, \\
\text { Ratio of }\end{array}$ & $\begin{array}{l}\text { Number of Special } \\
\text { Characters over Message } \\
\text { Length (ML) }\end{array}$ \\
\hline \#7 & $\begin{array}{l}\text { Punctuation } \\
\text { Characters (RPC) } \\
\text { such as "‘;?!: ( )- } \\
\text { " «» }<>[]\{\} "\end{array}$ & $\begin{array}{l}\text { Number of Punctuation } \\
\text { Characters over Message } \\
\text { Length (ML) }\end{array}$ \\
\hline \#8 & $\begin{array}{l}\text { Total number of } \\
\text { Digit Characters } \\
\text { (DC) }\end{array}$ & $\begin{array}{l}\text { Normalized by maximum } \\
\text { number of digit characters. }\end{array}$ \\
\hline \#9 & $\begin{array}{l}\text { The existence of } \\
\text { word "Call" and } \\
\text { digits together }\end{array}$ & $\begin{array}{l}\text { The value of this field is } \\
\text { either true or false }\end{array}$ \\
\hline \#10 & $\begin{array}{l}\text { The existence of } \\
\text { URL in the message }\end{array}$ & $\begin{array}{l}\text { The value of this field is } \\
\text { either true or false }\end{array}$ \\
\hline
\end{tabular}

\section{Feature Extraction}

In this research, in order to more concisely and accurately classify and detect SMS spam, unnecessary features were eliminated by using the consistency-based feature selection approach. In consistency-based feature selection, consistency measures were used to evaluate the importance of feature subsets. This measure is intuitively defined as a metric to measure the distance of a feature subset from the consistent state [19]. A feature set $\left\{F_{1} \ldots\right.$ $\left.F_{n}\right\}$ is said to be consistent, when Equation 1 holds for all $c, f_{1}, \ldots, f_{n}$.

$$
\operatorname{Pr}\left(C=c \mid F_{1}=f_{1}, \ldots, F_{n}=f_{n}\right)=0 \text { or } 1
$$

When a feature subset is consistent, the inconsistency value is 0 , and as an inconsistent feature subset approaches the consistent state, the measure decreasingly approaches 0 . To illustrate, $\{\mathrm{F} 1, \mathrm{~F} 2\}$ in our previous example is measured to be 0 , whereas the measure for $\{F 1\}$ and $\{F 2\}$ should be high [19].

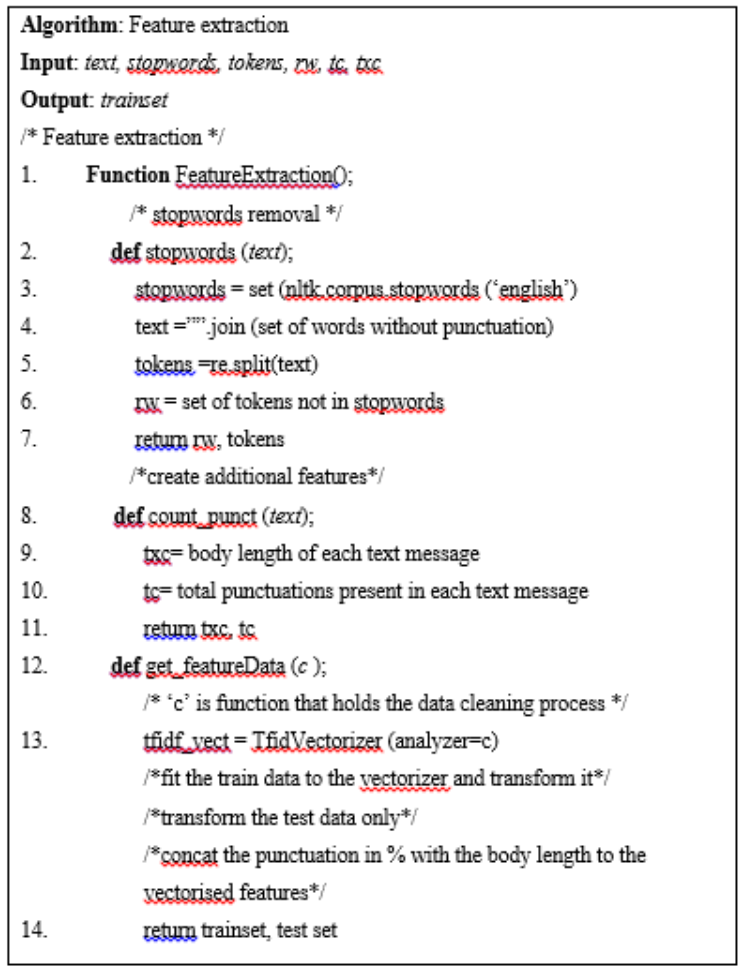

Fig.4. Feature Extraction Algorithm

The total number of characters in each column of the dataset was considered, the number of rows in the dataset and the numbers of punctuation that exist in the dataset. Features engineering was considered as the technique for extracting the features. Feature engineering was performed firstly by loading the SMS dataset, Performing feature engineering on the sms data by using the function data['body_len'] = data['body_txt'].apply(lambda $\mathrm{x}$ : len(x) - x.count(' ')), the next was to create feature for percentage of count of punctuation in the messages, to create additional features, data ['punct\%'] = data['body_txt'].apply(lambda x : create_punc_perct(x)) 
was applied. There are 10 features in the SMS dataset, all of these features were not relevant in the building of the model. Fig. 4 show the algorithm used to extract the features from the dataset.

\section{Ensemble Algorithm}

An ensemble method is a technique that combines the predictions from multiple machine learning algorithms together to make more accurate predictions than any individual model. In this research work, four machine learning algorithms were used: Random Forest (RF), Logistic Regression (LR), Multilayer Perceptron (MLP), and Support Vector Machine (SVM). They are described as follows:

1) Random Forest (RF) depends on a random selection of variables and data to develop a large number of decision trees. Random Forest uses decision trees to create bootstrap by selecting random features, it is considered as a special case from bagging. However, the main idea of RF is that Shallow trees which are called stumps will be pruned and tuned and the output after tuning and pruning will be aggregated, then RF will rely on such aggregation. The aggregation will lead to an accurate prediction by eliminating the error from trees.

2) Logistic Regression (LR) according to [13] is one of the most popular machine learning algorithms for binary classification. LR is also known as "logit" regression that is used for estimation of discrete value such as 0 and 1, true or false, yes and no, based on a set of variables. It performs better on varies of a solution and its predictive probability lies between 0 and 1 . This function is as follows in equation (2)

$$
T=\frac{1}{\left(1+e^{-x}\right)}
$$

$\mathrm{T}$ is the transformed, $e$ is the Euler's number, and $\mathrm{x}$ is the input that will be plugged into the function.

3) Multilayer perceptron (MLP) is a sub-class of an artificial neural network. An MLP comprises of, at least, three layers of nodes: an input, a hidden layer, and an output. Apart from the input nodes, every hub is a neuron that utilizes a nonlinear activation function. MLP uses a supervised learning system called back propagation for preparing. Its multiple layers and non-linear activation set MLP apart from a linear perceptron. It can distinguish data that is not linearly detachable. A multilayer perceptron is sometimes referred to as "vanilla" neural systems, particularly when they have a single hidden layer [14].

4) Support Vector Machine (SVM) is a machine learning method that is widely used for data analysis and pattern recognition. SVM has been very excellent in the case of classification. Classifying data has been one of the major parts of machine learning. The idea of support vector machine is to create a hyper plane in between data sets to indicate which class it belongs to. The challenge is to train the machine to understand the structure from data and mapping with the right class label, for the best result, the hyper plane has the largest distance to the nearest training data points of any class.

\section{B. Model Formulation}

The process involves detecting SMS spam was implemented using the process below and a class. The SMS spam system was developed using a python programming language in visual studio code environment, the data was preprocessed using Natural Language ToolKits (NLTK).

Additional features were created to improve the accuracy of the predicting machine. A total number of punctuations was calculated, and the total length of each word per records was calculated, and this step was done per each text message.

The two additional features added was "words length" and "number of punctuations" together with the vectorized text. Vectorization means turning the text into number since machine learning algorithms deal with a number and the library applied were TFidfVectorizer. The data were divided into training and test sets using the train_test_split library. All the algorithms received training sets of $80 \%$ and test sets $20 \%$

For cross-validation the whole datasets were slot into the machine, K-Fold algorithm was used, and $\mathrm{K}=10$. Therefore, the algorithm divides the dataset into 10-folds and 9-fold for training and 1-fold for testing, this was done until the whole folds pass through all phases. Each algorithm produces a model after slotting the training and test sets into the algorithms. Fig. 5 shows the algorithm used for the polarity detection from the user. The process starts by entering an SMS text message into the system. If the user does not enter a message the system alert that no imputed value into the system, the error tell the user to enter a text message. The text is cleaned in features sets and is fed into the classifiers. The text is classified based on the result of all the classifiers.

\section{RESULTS AND DISCUSSIONS}

For the purpose of this research, the simulation was carried out using a python programming language with its machine learning library (Scikit-learn library). The analysis of the simulation result and performance metrics of the formulated model are as follows:

\section{A. Simulation Results}

The dataset was divided into training and test sets using. All the machine learning algorithms received training sets of $80 \%$ and test sets $20 \%$. For crossvalidation the whole datasets were slot into the machine. Each algorithm produces a model after slotting the training and test sets into the algorithms. The developed system detects SMS spam messages. The user can enter 
an SMS message, the system analyzes the message and return spam or ham. Once the message is inputted, the text entered serves as a test to the models generated by each of the classifiers.

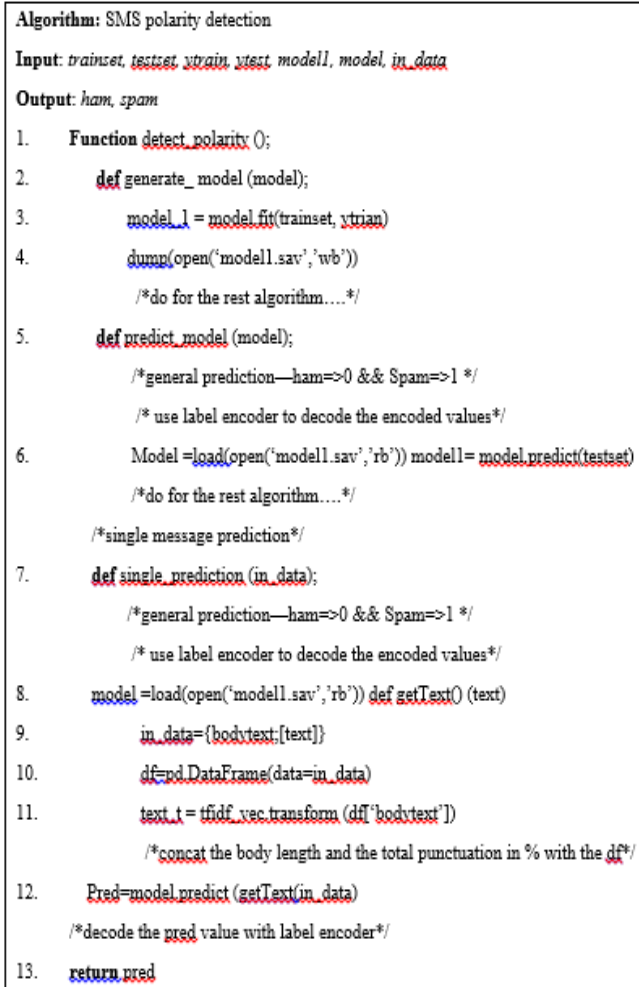

Fig.5. SMS Polarity detection

The system takes in an SMS message from the user, the features are extracted automatically by the system, the SMS and the accuracy of the message, are predicted by the model. Fig. 6 shows the screenshot for the extracted input, the extracted features show what the system uses to relate with the model for the system to detect the SMS spam. The user enters an SMS message in the system for example "To get 2.50 pounds free call credit and details of great offers pls reply 2 this text with your valid name, house no and postcode". The extracted features are fitted into the models. The result shows "Ham" which implies that the SMS message entered into the system is legitimate as shown in Fig. 7. To increase the performance of the system, the four classifiers were combined and predicted as a single result, for the inputted SMS message, the system gave a spam result as shown in Fig. 8. This implies that the sentence entered by the user is a spam message.

\section{B. Evaluation Results}

The performances of the algorithms (i.e. classifiers) were evaluated using Receiver Operation Characteristics (ROC) curve as the measure of text accuracy. The results in Table 3 and Fig. 9 showed that the selected classifiers perform well using the proposed model. Also, a simulation was carried out to ascertain the effectiveness of the ensemble method used. The result obtained in Table 4 and Fig. 10 established that the proposed model gives a good result with stack Generalization ensemble performing better than other ensembles such as VoltClassifier, Ada Boosting and Gradient Boosting. Performance evaluation of the proposed model was then carried out by benchmarking it with an existing model, H20 framework [4], using ROC, accuracy, precision, recall and F-measure as performance metrics. The result shown in Fig. 11 implied that the proposed model classifies accurately than the existing model. Also, the evaluation results in Table 5, shows that the proposed model gave an increase of $3.03 \%$ of accuracy, $8.94 \%$ of Recall, $2.17 \%$ of F-measure; and a decrease of $4.55 \%$ of Precision over the existing model. The result from the performance evaluation shows that there is an improvement in the accuracy of detecting SMS Spam.

Table 3. Receiver of operating characteristics for the classifiers

\begin{tabular}{lc}
\hline \multicolumn{1}{c}{ Classifiers } & Result \\
\hline Multilayer Perceptron & 96.89 \\
Support Vector Machine & 69.27 \\
Logistic Regression & 89.98 \\
Random Forest & 92.72 \\
\hline
\end{tabular}

Table 4. Ensemble technique evaluation

\begin{tabular}{lccccc}
\hline $\begin{array}{l}\text { Ensemble } \\
\text { Technique }\end{array}$ & $\begin{array}{c}\text { Accuracy } \\
\mathbf{( \% )}\end{array}$ & $\begin{array}{c}\text { Precision } \\
\mathbf{( \% )}\end{array}$ & $\begin{array}{l}\text { Recall } \\
\mathbf{( \% )}\end{array}$ & $\begin{array}{c}\text { F- } \\
\text { Measure } \\
(\%)\end{array}$ & ROC\% \\
\hline $\begin{array}{l}\text { Stack } \\
\text { Generalization }\end{array}$ & 98.03 & 91.46 & 94.94 & 93.17 & 96.74 \\
$\begin{array}{l}\text { VoltClassifier } \\
\text { Ada Boosting }\end{array}$ & 97.13 & 100 & 79.75 & 88.73 & 89.87 \\
$\begin{array}{l}\text { Gradient } \\
\text { Boosting }\end{array}$ & 97.13 & 88.41 & 91.77 & 90.06 & 94.89 \\
\hline
\end{tabular}

Table 5. Table Performance Evaluation results

\begin{tabular}{lllll}
\hline S/N & Metrics & $\begin{array}{l}\text { Existing } \\
\text { Model } \\
{[\mathbf{4}]}\end{array}$ & $\begin{array}{l}\text { Proposed } \\
\text { Model }\end{array}$ & $\begin{array}{l}\text { Percentage } \\
\text { difference }\end{array}$ \\
\hline $\mathbf{1}$ & ACCURACY & $95 \%$ & 98.03 & $3.03 \%$ \\
$\mathbf{2}$ & PRECISION & $96 \%$ & $91.46 \%$ & $4.55 \%$ \\
$\mathbf{3}$ & RECALL & $86 \%$ & $94.94 \%$ & $8.94 \%$ \\
$\mathbf{4}$ & F-MEASURE & $91 \%$ & $93.17 \%$ & $2.17 \%$ \\
$\mathbf{5}$ & ROC & & $97.74 \%$ & \\
\hline
\end{tabular}




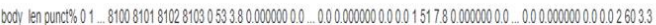

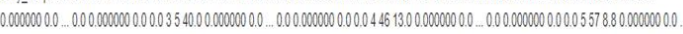

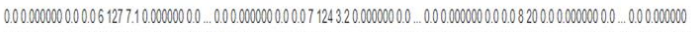
$0.00 .092441 .702848380 .0 .0 .0 .0000000 .00 .010238 .70 .3733100 .0 \ldots 000.0000000 .00 .011640 .00 .1744150 .0 .0 .00 .0000000 .00 .01228$ 7.1000000000000000000000000131246500000000000000000000000142442000000000000000000000 .00 .01524250

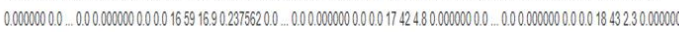

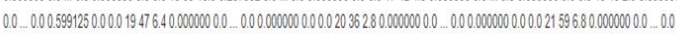

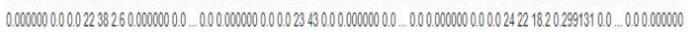

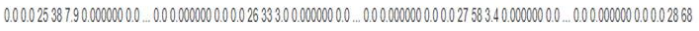
$13200000000000000000000002913151000000000000000000000.000 \quad 4423125880000000000$

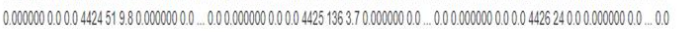

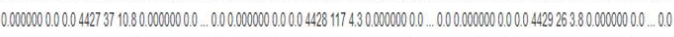

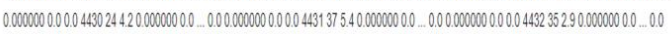

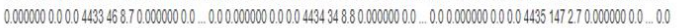
0.000000000004436231740433932000000000000000044374424000000000000000000000004438660000000000000 .00

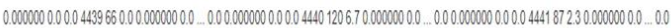

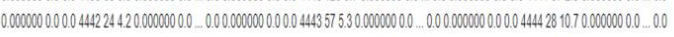

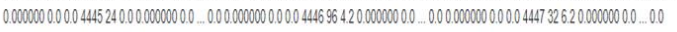
0.000000000004448132830000000000 .00000000000000444966450000000000 . 00000000000000445088102000000000

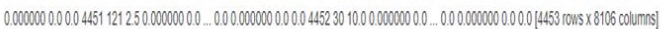

Fig.6. Extracted input features

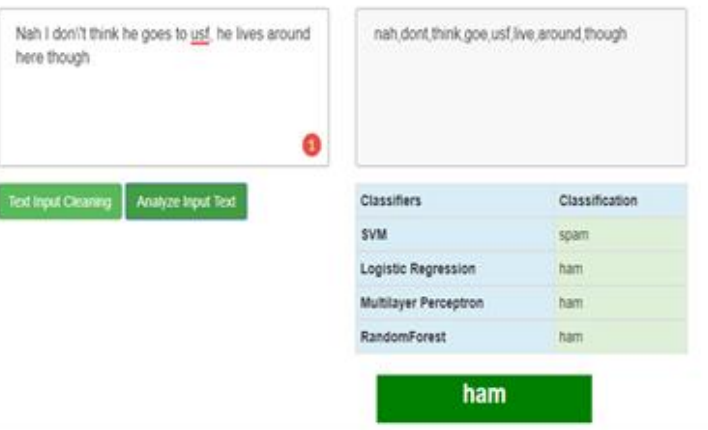

Fig.7. SPAM detection result

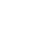

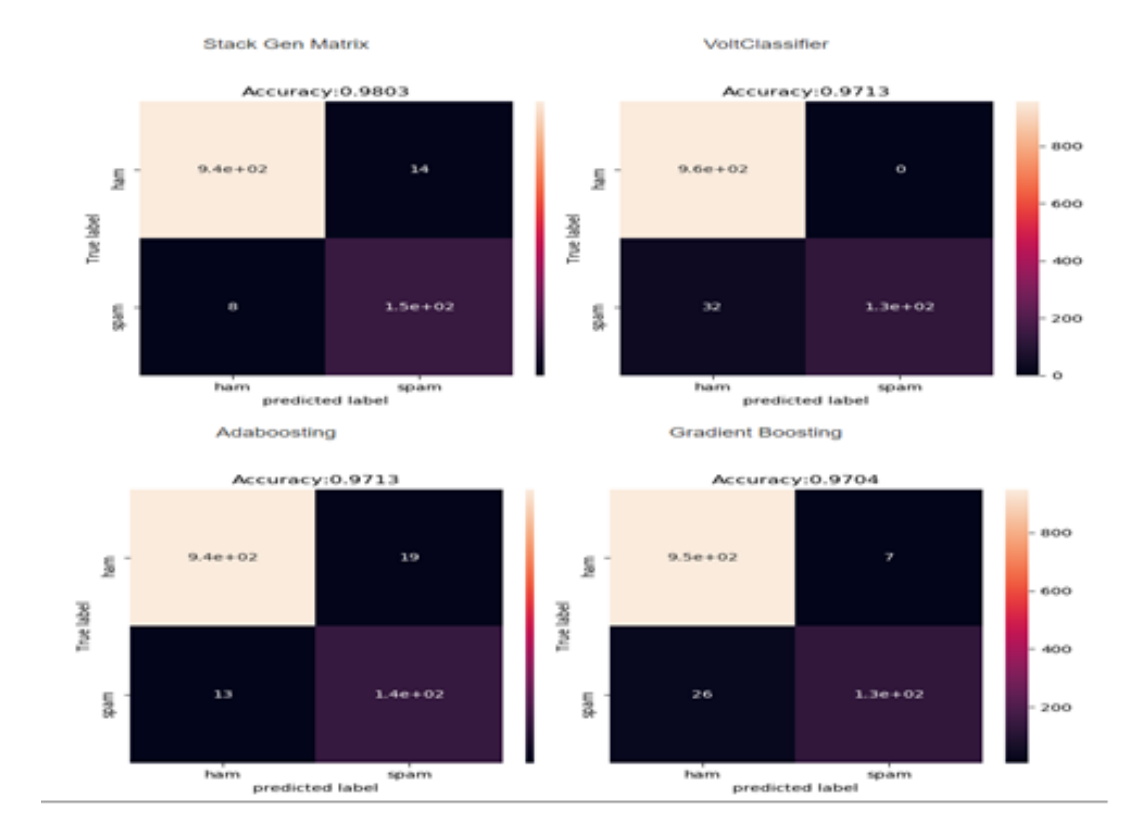

Fig.10. Confusion Metrics of the Ensemble Algorithms

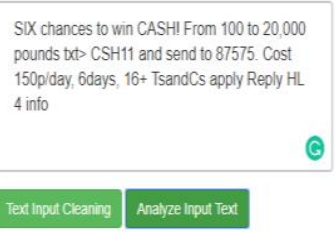

six chanc, win, cash, 100,20000 . pound txt. csh 11 , end, 87575 , cost, 150pday, 6 day, 16 , tsandc appli, epli,il, 4 , info

\section{(2)}

\begin{tabular}{ll}
\hline Classifiers & Classification \\
\hline SVM & spam \\
\hline Logistic Regression & spam \\
\hline Muttlayer Perceptron & spam \\
\hline RandomForest & spam \\
\hline
\end{tabular}

spam

Fig.8. SPAM detection using Ensemble Techniques

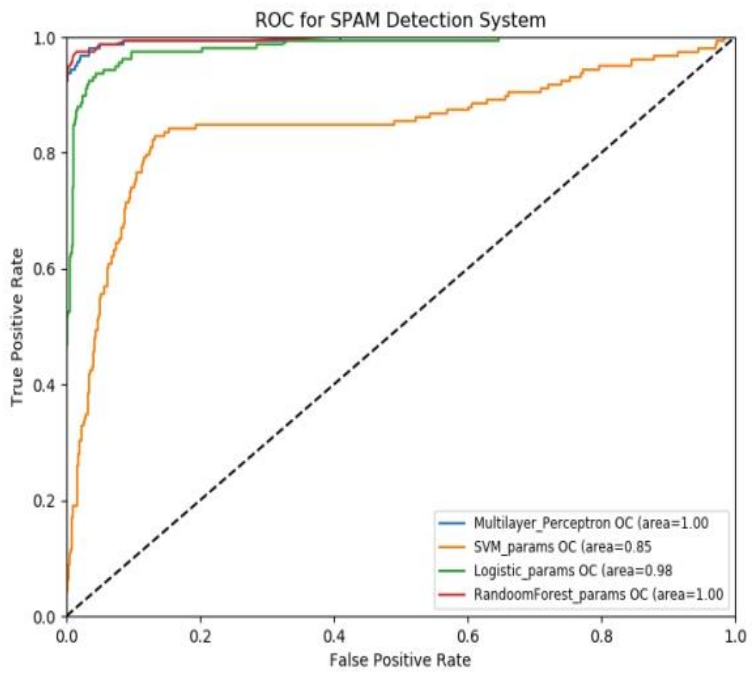

Fig.9. The ROC curve of the classifier 


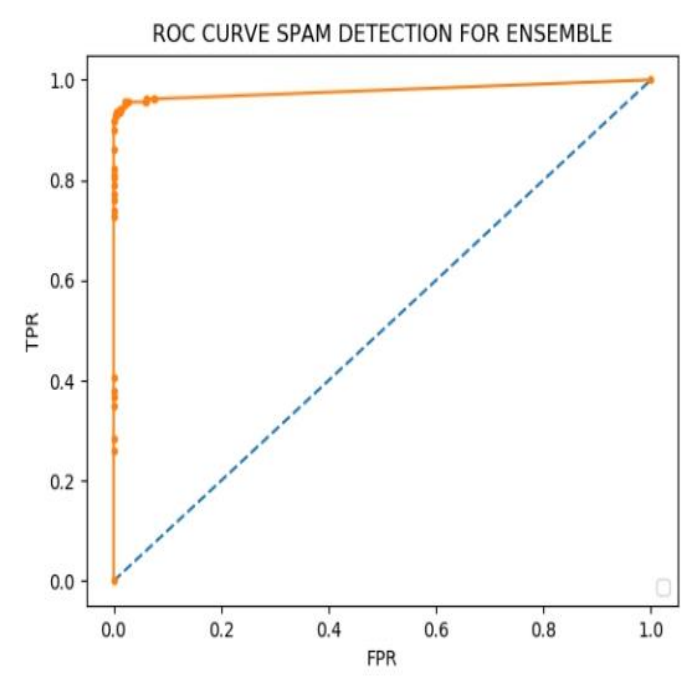

Fig.11. The ROC curve of the Existing and Proposed model

\section{CONCLUSION}

SMS is one of the best mediums of communication amongst mobile user, they are of two types: ham and spam, spam message are the annoying and unfortunate messages that must be evacuated or obstructed before the client getting them. An SMS spam filtering framework was developed using an ensemble of four machine learning algorithms namely, Random Forest (RF), Logistic Regression (LR), Multilayer Perceptron (MLP), and Support Vector Machine (SVM). The proposed model was simulated and it was obvious in the results that the proposed model has a high reduction in the instances of false negatives states and high detection accuracy, sensitivity and precision rates respectively, thus has a better performance in detecting spam and ham messages. Therefore, the proposed model can be adapted by the network service providers for better Quality of Service (QoS). The current framework requires that the dataset of the model is specified explicitly, the future works is directed to having a model that will allow automatic learning of the structure of the SMS spam detection model.

\section{ACKNOWLEDGMENTS}

This Research was funded by the TETFund Research Fund" and Africa Centre of Excellence OAK-Park, Obafemi Awolowo University, Ile-Ife, Nigeria.

\section{REFERENCES}

[1] A. Al-Hassana, E. M. El-Alfyb, "Dendritic Cell Algorithm for Mobile Phone Spam Filtering," 6th International Conference on Ambient Systems, Networks and Technologies, Procedia Computer Science, vol. 52, pp. $244-251,2015$.

[2] Baldwin, "350,000 different types of spam SMS messages were targeted at mobile users in 2012," Computer weekly publication [online] February 2013. Available: https://www.computerweekly.com/news/2240178681/350 000-different-types-of-spam-SMS-messages-were- targeted-at-mobile-users-in-2012

[3] D.N. Sohn, J.T. Lee, K.S. Han, and H.C. Rim, "Contentbased mobile spam classification using stylistically motivated features". Pattern Recognition Letters, vol. 33, no. 3, pp.364-369, 2012.

[4] Suleiman and G. Al-Naymat, "SMS Spam Detection Using H2O framework." Procedia Computer Science, vol. 113, pp 154-161, 2017.

[5] H. Sajedi, G. Z. Parast, and F. Akbari, "SMS Spam Filtering Using Machine Learning Techniques: A Survey" . Machine Learning Research. Vol. 1, no. 1, pp. 14, 2016.

[6] N. Choudhary and A.K.Jain. "Towards Filtering of SMS Spam Messages Using Machine Learning Based Technique". In: Singh D., Raman B., Luhach A., Lingras P. (eds) Advanced Informatics for Computing Research. Communications in Computer and Information Science, Springer, Singapore, vol. 712, pp 18-30, 2017.

[7] L. N. Lota and B M Mainul Hossain ,"A Systematic Literature Review on SMS Spam Detection Techniques", International Journal of Information Technology and Computer Science (IJITCS), vol.9, no.7, pp.42-50, 2017

[8] T.H. Pham and P. Le-Hong, "Content-based Approach for Vietna- mese Spam SMS Filtering". In proceedings of 2016 International Conference on Asian Language Processing (IALP), Tainan, pp. 41-44, 2016.

[9] G.V. Cormack, J.M. Gómez Hidalg, and E.P. Sánz, "Feature Engineering for mobile (SMS) spam filtering," Proceedings of the 30th annual international ACM SIGIR conference on Research and development in information retrieval, July 23- 27, 2007, Amsterdam, pp 871-872, 2007.

[10] N. Chaudhari, P. Jayvala, and P. Vinitashah," Survey on Spam SMS filtering using Data mining Techniques," International Journal of Advanced Research in Computer and Communication Engineering, Vol. 5, Issue 11, 2016

[11] I. Ahmed, D. Guan and T. C. Chung, " SMS Classification Based on Naïve Bayes Classifier and Apriori Algorithm Frequent Itemset,“ International Journal of Machine Learning and Computing, Vol. 4, No. 2, pp 184-187, 2014

[12] K. Yadav, P. Kumaraguru, A. Goyal, A. Gupta and V. Naik, "SMS Assassin: Crowdsourcing Driven Mobilebased System for SMS Spam Filtering," in Proceedings of the 12th Workshop on Mobile Computing Systems and Applications, pp 1-6, 2011.

[13] J. Brownlee, "Machine Learning Mastery with Python: Understand Your Data, Create Accurate Models and Work Projects End-To-End., Edition: v1.5, pp 1-24, 2016,

[14] H. Trevor, T. Robert, J. H Friedman and F. James, "The Elements of Statistical Learning: Data Mining, Inference, and Prediction," In proceedings of the Mathematical Intelligencer, Vol. 27, No 2, pp 83-85, 2004.

[15] T. A. Almeida and J. M Gómez Hidalgo, "SMS Spam Collection Data Set- UCI Machine Learning Repository," Available:

https://archive.ics.uci.edu/ml/datasets/SMS+Spam+Collect ion. 2011

[16] S. Guido and A. C. Muller, "Introduction to machine learning with Python: a guide for data scientists. O'Reilly Media, Inc., 2016

[17] H. Shirani-Mehr, "SMS Spam Detection using Machine Learning Approach," CS229 Project 2013, Stanford University, USA, pp. 1-4, 2013

[18] S. Schrauwen, "Machine learning approach to sentiment analysis using the Dutch Netlog Corpus." Computational Linguistic and Psycholingistics Research Center, pp1-78, 
2010

[19] K. Shin, D. Fernandes and S. Miyazaki. "Consistency Measure for feature Selection: A formal Definition, Relative Sensitivity Comparison and a fast Algorithm". In Proceeding of Twenty -Second International Joint Conference on Artificial Intelligence, pp 1491-1497, 2011

\section{Authors' Profiles}

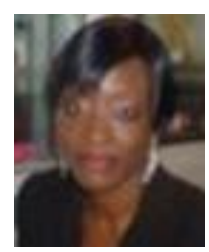

ODUKOYA OLUWATOYIN is a Lecturer I at Obafemi Awolowo University, Ile-Ife. Her research interests are Cybersecurity, Data mining and Data Communication and Networking (DCN).

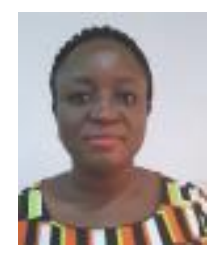

AKINYEMI Bodunde is a Senior Lecturer at Obafemi Awolowo University, Ile -Ife. Her current research interest include CyberSecurity, Data communication and networking. She is a Member of the Nigeria Computer Society (MNCS) and a chartered IT practitioner (MCPN).

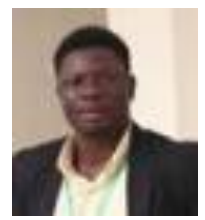

GOODING TITUS is a post-graduate student of Obafemi Awolowo University, Ile-Ife, Nigeria. His current research interest include cyber security and Data mining.

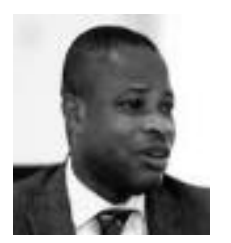

ADEROUNMU Ganiyu is a professor at Obafemi Awolowo University, Ile-Ife, Nigeria. He is a Full member of the Nigeria Society of Engineers (FNSE), Nigeria Computer Society (FNCS), a chartered IT practitioner (CPN) and certified Engineer (COREN).

How to cite this paper: Odukoya Oluwatoyin, Akinyemi Bodunde, Gooding Titus, Aderounmu Ganiyu, "An Improved Machine Learning-Based Short Message Service Spam Detection System", International Journal of Computer Network and Information Security(IJCNIS), Vol.11, No.12, pp.40-48, 2019. DOI: 10.5815/ijenis.2019.12.05 